\title{
Renbök phenomenon: Alopecia areata sparing psoriasis plaques
}

\section{Mrinal Gupta, Vikram K Mahajan}

\author{
Department of Dermatology, Venereology \& Leprosy, Dr. R. P. Govt. Medical College, Kangra (Tanda)-176001 (Himachal \\ Pradesh), India
}

Corresponding author: Dr. Vikram K Mahajan, E-mail: vkm1@rediffmail.com

Sir,

Researchers have tried to elucidate the poorly understood mechanisms underlying localization of skin lesions leading to dermatoses-specific clinical presentations from some interesting phenomena such as of "co-localization" or "co-existence", "Wolf's isotopic phenomenon", "Koebner's (isomorphic) phenomenon" and recently described "Renbök phenomenon" having only few cases reported as yet.

A 45- year-old man presented with progressively increasing asymptomatic patchy scalp hair loss of sudden onset for one month. He had plaque psoriasis involving scalp and extensors for the last 7 years with infrequent relapses. The lesions used to clear following treatment with topical coal tar $3 \%$ solution and shampoo. His family and medical history, and laboratory investigations (complete blood counts, hepatorenal and thyroid function tests, anti-thyroid and antinuclear antibody profile) were unremarkable. Cutaneous examination showed psoriatic plaques localized over vertex and fronto-temporal region and multiple patches of alopecia areata involving the occipital scalp (Fig. 1). The psoriasis plaques were well delineated, confluent and stopped short of alopecia areata patches without any encroachment and showed normal hair growth. The hair loss in patches of alopecia areata was total and spared psoriatic plaques reflecting Renbök phenomenon. He did not consent for skin biopsy. He was prescribed twice daily topical application of betamethasone dipropionate $0.05 \%$ lotion and to follow-up regularly.

The phenomenon of "co-localization" or "coexistence" of psoriasis or alopecia areata appearing

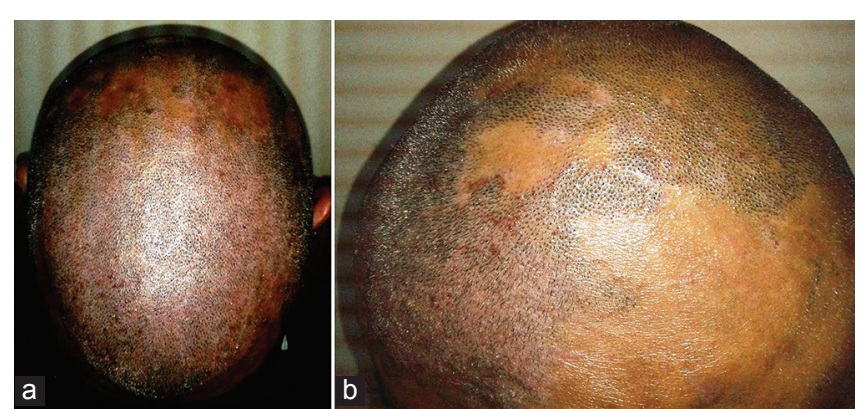

Figure1: (a) Psoriasis lesions with normal hair growth and involving vertex and fronto-temporal scalp stopping just short of alopecia patches. (b) Patches of alopecia areata devoid of hair present over occipital scalp sparing psoriasis lesions.

over vitiligo-affected skin is not uncommon. While structural similarities between anti-stratum corneum antibodies and anti-melanocyte antibodies, and a common neuropeptide have been speculated possible causative factors for co-localization of psoriasis on vitiligo, the melanocyte-derived antigens (melanocyte epitopes) released in pathogenesis of vitiligo are postulated to act like auto-antigens inducing hair loss in co-localizing alopecia areata [1]. The "Wolf's isotopic phenomenon" describes occurrence of a new skin disease at the site of another, unrelated and already healed skin disease and is different from "isomorphic response" described by Koebner as early as 1876 [2]. The "Koebner's (isomorphic) phenomenon" means appearance of clinicopathologically identical skin lesions of an existing dermatosis at the site of injury [3]. While psoriasis remains its classic prototype, this phenomenon also characterizes lichen planus as well as vitiligo. Interestingly, Koebner's phenomenon of psoriasis aggravation over sites of alopecia areata too has been described [4]. Its

\footnotetext{
How to cite this article: Gupta M, Mahajan VK. Renbök phenomenon: Alopecia areata sparing psoriasis plaques. Our Dermatol Online. 2018;9(3):333-334. Submission: 06.11.2017; Acceptance: 13.01.2018

DOI:10.7241/ourd.20183.28
} 
pathogenetic mechanisms are poorly understood and immunologic, vascular, dermal, enzymatic, inhibitory, neural, growth, genetic and hormonal factors have been implicated [3]. Contrarily, inverse of Koebner's phenomenon is seen when an area of psoriasis clears or re-pigmentation of vitiligo lesion occurs following trauma (electrodessication, dermabrasion, surgery) [5].

Renbök phenomenon, a reverse of 'Koebner' phenomenon, is a relatively recent term coined by Happle et al [6]. It was considered peculiar to patients with psoriasis having alopecia areata wherein hair growth over psoriatic plaques is normal [7]. Later, its application was expanded to include alopecia areata similarly sparing nevus flammeus or congenital nevus [4]. Its pathomechanism is unknown and it has been hypothesized that in psoriasis (Th-17 mediated dermatoses) and alopecia areata (Th-l mediated disorder) T-cell subsets by cytokine production enhance their own response through positive feedback while antagonizing the other responses in the affected areas $[4,8]$. Hence, only a single subset of inflammatory response is seen at a particular site. Criado et al [7] also proposed that the microenvironment of high levels of TNF- $\alpha$ in psoriasis is not favorable for the development of inflammatory response in alopecia areata producing characteristic Renbök phenomenon. However, its exact pathogenesis remains poorly elucidated for paucity of reported cases.

\section{CONSENT}

The examination of the patient was conducted according to the Declaration of Helsinki principles.

\section{REFERENCES}

1. Rodriguez-Martin M, Merino N, Contreras P, Santana SS, Martín BR, Martin-Herrera A. Anatomical colocalization of vitiligo and alopecia areata. Open Autoimmun J. 2010;2:193-6.

2. Sharma RC, Sharma NL, Mahajan V, Sharma A. Wolf's isotopic response: herpes simplex appearing on scrofuloderma scar. Int J Dermatol. 2003;42:664-6.

3. Thappa DM. The isomorphic phenomenon of Koebner. Indian J Dermatol Venereol Leprol. 2004;70:187-9.

4. Harris JE, Seykora JT, Lee RA. Renbök phenomenon and contact sensitization in a patient with alopecia universalis. Arch Dermatol. 2010;146:422-5.

5. Oripelaye MM, Onayemi O, Olasode OA, Olanrewaju FO. Vitiligo on tribal mark: A demonstration of Wolf's isotopic response. Our Dermatol Online. 2017;9:48-50.

6. Happle R, van der Steen PHM, Perret CM. The Renbok phenomenon: An inverse Koebner reaction observed in alopecia areata. Eur J Dermatol. 1991;2:39-40.

7. Criado PR, Valente NY, Michalany NS, Martins JEC, Romiti R, Aoki V, et al. An unusual association between scalp psoriasis and ophiasic alopecia areata: the Renbök phenomenon. Clin Exp Dermatol. 2007;32:320-1.

8. Kannangara AP, Fleischer AB, Yosipovitch G. The sparing phenomenon. A case series of the inverse Koebner and related phenomena. Our Dermatol Online. 2013;4: 35-9.

Copyright by Mrinal Gupta, et al. This is an open-access article distributed under the terms of the Creative Commons Attribution License, which permits unrestricted use, distribution, and reproduction in any medium, provided the original author and source are credited.

Source of Support: Nil, Conflict of Interest: None declared. 